\title{
Dose-escalated radiotherapy improved survival for esophageal cancer patients with a clinical complete response after standard-dose radiotherapy with concurrent chemotherapy
}

This article was published in the following Dove Press journal: Cancer Management and Research

\author{
Wei Zhangl,* \\ Yijun Luo ${ }^{2, *}$ \\ Xiaoli Wang ${ }^{2}$ \\ Gaohua Han' \\ Peng Wang' \\ Wei Yuan ${ }^{3}$ \\ Sheng-Bin Dai' \\ 'Department of Oncology, Taizhou \\ People's Hospital, Taizhou, Jiangsu, \\ People's Republic of China; \\ 2Department of Oncology, The \\ People's Hospital of Jiangxi Province, \\ Nanchang, Jiangxi, People's Republic \\ of China; ${ }^{3}$ Department of Cardiology, \\ Taizhou People's Hospital, Taizhou, \\ Jiangsu, People's Republic of China \\ *These authors contributed equally to \\ this work
}

Correspondence: Wei Yuan

Department of Cardiology, Taizhou

People's Hospital, South Hailing Road

399, Taizhou 225300, People's Republic of China

Tel +865238660677l

Email yuanweiht@I26.com

Sheng-Bin Dai

Department of Oncology, Taizhou

People's Hospital, South Hailing Road

399, Taizhou 225300, People's Republic

of China

Tel +8652386606004

Email13515155717@163.com
Purpose: For esophageal cancer patients with a clinical complete response (cCR) after standarddose radiotherapy with concurrent chemotherapy, data on the survival outcomes and recurrence patterns remain scarce. To evaluate the impact of dose escalation on overall survival for this subset of patients, we carried out the current investigation.

Materials and methods: Medical records of 80 esophageal cancer patients with a cCR after standard-dose radiotherapy with concurrent chemotherapy at our center from 2010 to 2014 were allocated into the standard-dose group (50.4 Gy, observation group) or the radiation dose-escalation group (59.4 Gy, control group). In this cohort study, we compared the outcomes between the 2 groups.

Results: There were no differences in patient characteristics between the 2 groups. The median recurrence-free survival and overall survival times for all patients were 38 and 54 months, respectively. Patients in the control group had significantly better 5 -year recurrence-free survival rate $(12 \%$ vs $0 \%, p=0.019)$ and 5 -year overall survival rate $(42.8 \%$ vs $21.0 \%, p=0.028)$ than the observation group. Additionally, local control rate was significantly higher in the control group ( $p=0.04$ ), and $\sim 60 \%$ of treatment failures were local failures even for patients achieving cCR after chemoradiotherapy. There were no significant differences in treatment-related toxicities between the groups.

Conclusion: The results of the current study suggest that for esophageal cancer patients with a cCR after standard-dose radiotherapy with concurrent chemotherapy, those with dose-escalated radiotherapy showed significantly better local control, recurrence-free survival, and overall survival than patients receiving 50.4 Gy radiotherapy.

Keywords: esophageal squamous cell carcinoma, radiotherapy, survival outcome, recurrence patterns

\section{Introduction}

Esophageal cancer (EC) is one of the most common cancers worldwide and has a poor prognosis due to high rates of local recurrence (LR) and distant metastasis. ${ }^{1,2}$ Squamous cell carcinoma is the most common histology of EC in Asia. ${ }^{3}$ About one-half of patients presented with locally advanced stage at the time of diagnosis. ${ }^{4}$

Definitive chemoradiotherapy (CRT) has been recommended as the optimal treatment for patients who are medically inoperable or have locally advanced stage tumor based on the results of the Radiation Therapy Oncology Group (RTOG) 85-01 trial. ${ }^{5}$ Despite curative treatments, the 5-year overall survival (OS) rate for esophageal 
squamous cell carcinoma (ESCC) patients is $\sim 20 \%{ }^{6}$ The importance of optimizing radiation dose for definitive management is particularly high for EC since treatment outcomes remain poor. Attempts to improve OS by escalating the dose of radiotherapy with concurrent chemotherapy has been assessed in the INT 0123 trial. $^{7}$ OS after 64.8 Gy dose was not superior or even lower to the 50.4 Gy. Based on those results, NCCN esophageal cancer guidelines recommend radiation doses of 50.4 Gy for definitive concurrent CRT (CCRT) in EC.

However, because of high local failure and distant failure rates after treatment, new approaches such as intensification of CRT and escalation of the radiation dose have been attempted. ${ }^{8-10}$ Some studies have revealed that high radiation doses can bring survival benefits. However, some studies showed that dose-escalated radiotherapy do not confer a benefit to EC patients and can even lead to more treatmentrelated toxicities. Of note, patients with ESCC receiving CRT seem to have a disparity in treatment response and outcomes. For radiosensitive EC, the standard radiation dose of 50.4 Gy is sufficient, while for the resistant patient, it is difficult to achieve a complete response (CR) even via radiation dose escalation, which may further increase treatment-related toxicities. Ma and colleagues declared that for patients with $\mathrm{CR}$, the standard radiation dose of $50.4 \mathrm{~Gy}$ is sufficient and that these patients may not need to receive dose escalation after the standard-dose radiotherapy. ${ }^{11}$ However, a great number of studies demonstrated that local control after definitive CRT for EC remains a problem and that most local failures occur within the gross tumor volume (GTV), even for cases with a CR after CRT. ${ }^{12-14}$ A study by Di Fiore et $\mathrm{al}^{12}$ also showed that for the 86 clinical CR patients after CRT, 34 (39.5\%) experienced a local disease recurrence, 37 patients (43\%) experienced metastatic disease, and 19 of them experienced both of these. Other studies have come to the same conclusion. ${ }^{13,14}$ These data indicate that the standard dose (50.4 Gy in 28 fractions) may be inadequate to achieve a high probability of local control even for cases with a CR after CRT.

To the best of our knowledge, data on the survival outcomes and recurrence patterns of EC patients who achieved a cCR after CRT remain scarce. The purpose of our study was to evaluate and compare the oncologic outcomes of EC patients who, after treatment with standard-dose radiotherapy (50.4 Gy in 28 fractions), achieved a cCR and were then managed according to a wait-and-see policy (observation group) or escalated with higher dose radiotherapy (control group).

\section{Materials and methods}

\section{Patient selection}

We retrospectively reviewed the records of patients with EC who underwent CRT in 2 hospitals between January 2009 and March 2014. The criteria for enrollment were as follows: 1) All the patients had histologically proven primary ESCC and were treated with definitive CRT, and 2) only patients who achieved a CR after planned radiation (50.4-56 Gy) were included in the study. In addition, we excluded patients with thoracic esophageal squamous cell carcinoma who did not receive sufficient CCRT doses ( $<50.4$ Gy). Ultimately, 80 patients who achieved a cCR were recruited for this study, and we categorized them into 2 groups based on the radiation doses to compare their treatment outcomes: group 1 (patients receiving CCRT <56 Gy) and group 2 (patients receiving CCRT $\geq 59.4$ Gy). The median total dose and fraction size of RT in groups 1 and 2 were 52 Gy (50.4-56 Gy, fraction size: 1.80/2.0 Gy) and 62.0 Gy (59.4-64.8 Gy, fraction size: $1.80 / 2.0$ Gy), respectively. The study selection process is summarized in Figure 1.

CR for the primary tumor was defined as the absence of evidence of a residual tumor on esophageal endoscopy; on computed tomography (CT) of the neck, chest, and abdomen; and on endosonography of the esophagus. CR for lymph nodes was defined according to the Response Evaluation Criteria in Solid Tumors guidelines. Pretreatment evaluations included barium tests; esophageal endoscopy; CT of the neck, chest, and abdomen; and endosonography of the esophagus. Patients were staged according to the 2010 (7th) American Joint Committee on Cancer staging criteria. Tumor length was measured by esophagography before treatment. This study was approved by the Institutional Review Board of Taizhou People's Hospital (IRB 20170401) and the People's Hospital of Jiangxi Province (IRB160815), and all patients had signed written informed consent prior to the study.

\section{CRT}

Radiotherapy was administered as 3-dimensional conformal radiotherapy or intensity-modulated radiation therapy. A conventional fractionation schedule (daily 1.8-2.0 Gy per fraction, 5 days per week) was used in all patients. The delineation of clinical target volumes was based on CT, barium esophagogram, endoscopic examination, and positron emission tomography (PET) imaging. The clinical target volumes (CTVs) were a $3-\mathrm{cm}$ proximal and distal margin, and a $0.5-0.8-\mathrm{cm}$ radial margin was added to the GTV. The planning target volume (PTV) encompassed a $1-\mathrm{cm}$ proximal 


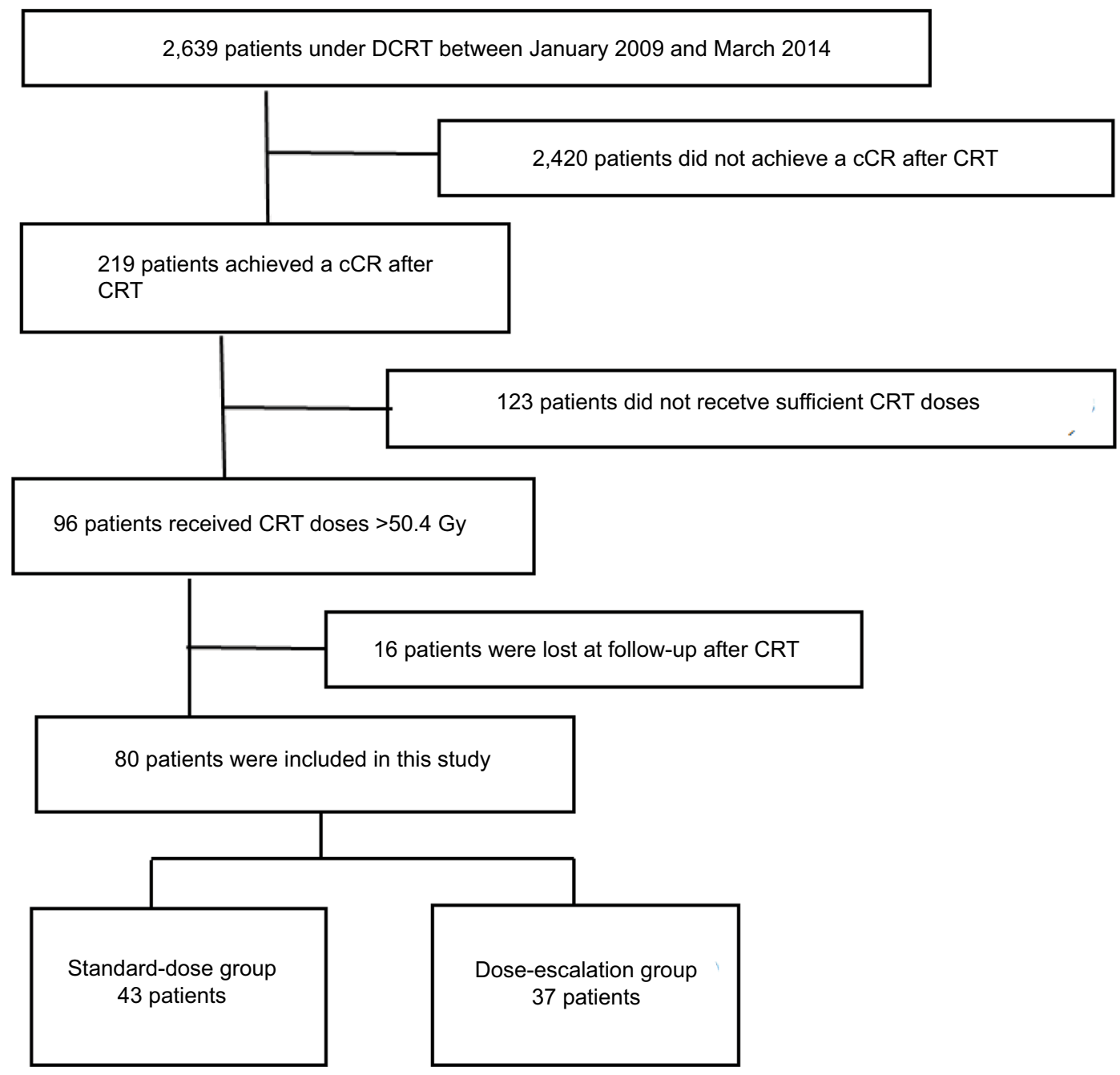

Figure I Study flow diagram.

Abbreviations: DCRT, definitive chemoradiotherapy; CCR, clinical complete response; CRT, chemoradiotherapy.

and distal margin and a $0.5-\mathrm{cm}$ radial margin based on CTVs. The nodal CTV was defined by a $0.5-1-\mathrm{cm}$ expansion around the nodal GTV. The PTV was the CTV plus a uniform $0.5-\mathrm{cm}$ expansion margin. In the dose-escalation group, 37 patients with $\mathrm{CR}$ received dose escalation after standard radiotherapy (50.4 Gy), the escalated dose was from 9 to $14.4 \mathrm{~Gy}$, and the total dose was ranged from 59.4 to $64.8 \mathrm{~Gy}$.

All patients received platinum-based chemotherapy concurrently with irradiation. The additional drug was either a 5-fluorouracil or taxane (docetaxel or paclitaxel).

\section{Definition of event}

Treatment-induced toxicities were assessed using the National Cancer Institute Common Terminology Criteria for Adverse Events (version 4.0). Severe adverse events were defined as toxicity grade $\geq 3$. Survival analyses were performed for recurrence-free survival (RFS) and OS. OS was measured from the first day of treatment to the date of death from any cause or the last follow-up, whichever occurred first. RFS was defined as the first evidence of any treatment failure. The patterns of treatment failure were defined according to the initial site of recurrence. Locoregional recurrence was defined as a recurrence occurring in the radiation field. Recurrences at any other sites were considered as distant recurrences.

\section{Follow-up}

All patients were examined weekly during RT to monitor treatment toxicities and their general condition. Routine evaluations included physical exam, hematologic and biochemical profiles, and esophagography. Follow-up evaluations were performed after the completion of all treatment, every 3 months for 2 years, and then every 6 months thereafter. The follow-up evaluations included physical examination, esophagography, esophagogastroduodenoscopy, chest and abdominal CT, and 
PET when available. Other necessary examinations were conducted according to the clinical situation.

\section{Statistical analysis}

All statistical analyses were carried out using SPSS (version 20.0; IBM Corporation, Armonk, NY, USA). $\chi^{2}$ test was performed to compare the difference of patients' characteristics. The rates of OS and RFS were calculated by the Kaplan-Meier method, with comparison using a log-rank test. All statistical tests were 2 -tailed, and $p<0.05$ was considered statistically significant.

\section{Results}

\section{Patient characteristics}

The patient characteristics are shown in Table 1. In total, $58.8 \%$ of patients were male, with a median age of 60 , while $41.2 \%$ were female, with a median age of 67.5 , and 43 patients were in the standard-dose group and 37 patients were in the dose-escalation group. Notably, more patients with stage III disease were included in the high-dose group, although the difference was not significant ( $62.2 \%$ vs $46.5 \%$, $p=0.162$ ). The median follow-up exceeded 54 months, with a maximum follow-up of 91 months. Patients in the 2 groups were similar with respect to age, sex, Karnofsky performance status, tumor location, tumor length, and clinical stage ( $p>0.05$ in all cases).

\section{Survival}

The median RFS of the patients in the conventional group and dose-escalation group were 35 and 44 months, respectively. Also, the 3- and 5-year RFS rates were $70.3 \%$ and $12 \%$ in the radiation dose-escalation group, which were significantly

Table I Patient, disease, and treatment characteristics

\begin{tabular}{|c|c|c|c|c|}
\hline Characteristics & All patients (\%) & $\begin{array}{l}\text { Standard-dose group } \\
\text { (50.4-56 Gy) }\end{array}$ & $\begin{array}{l}\text { Dose-escalation group } \\
(\geq 59.4 \mathrm{~Gy})\end{array}$ & $p$-value \\
\hline Age at diagnosis (years) & & & & - \\
\hline Median (range) & 67 (46-79) & 67 (48-79) & 68 (46-79) & \\
\hline Sex & & & & 0.905 \\
\hline Male & 47 & 25 & 22 & \\
\hline Female & 33 & 18 & 15 & \\
\hline Smoking at diagnosis & & & & 0.832 \\
\hline No & 25 & 13 & 12 & \\
\hline Yes & 55 & 30 & 25 & \\
\hline KPS & & & & 0.884 \\
\hline $90-100$ & 59 & 32 & 27 & \\
\hline$\leq 80$ & 21 & 11 & 10 & \\
\hline Tumor location & & & & 0.939 \\
\hline Proximal & 14 & 8 & 6 & \\
\hline Middle & 31 & 16 & 15 & \\
\hline Distal & 35 & 19 & 16 & \\
\hline Tumor length (cm) & & & & 0.666 \\
\hline$\leq 5$ & 41 & 23 & 18 & \\
\hline$>5$ & 39 & 20 & 19 & \\
\hline Clinical stage & & & & 0.162 \\
\hline II & 37 & 23 & 14 & \\
\hline III & 43 & 20 & 23 & \\
\hline RT technique & & & & 0.770 \\
\hline 3D-CRT & 36 & 20 & 16 & \\
\hline IMRT & 44 & 23 & 21 & \\
\hline Recurrence & & & & 0.487 \\
\hline Yes & 55 & 31 & 24 & \\
\hline No & 25 & 12 & 13 & \\
\hline Death & & & & 0.314 \\
\hline Yes & 48 & 28 & 20 & \\
\hline No & 32 & 15 & 17 & \\
\hline Chemotherapy & & & & 0.666 \\
\hline Cisplatin + paclitaxel & 39 & 20 & 19 & \\
\hline Cisplatin + FU/S-I & 41 & 23 & 18 & \\
\hline Total patients & 80 & 43 & 37 & \\
\hline
\end{tabular}

Abbreviations: KPS, Karnofsky performance score; 3D-CRT, 3-dimensional conformal radiotherapy; IMRT, intensity-modulated radiotherapy; RT, radiotherapy. 
higher than those in the standard-dose group ( $42 \%$ and $0 \%$, $p=0.019$ ).

The median OS was 48 months (conventional group) and 59.8 months (dose-escalation group) for the 2 groups, respectively. The 3- and 5-year OS rates were $75.3 \%$ and $21 \%$ for patients in the conventional group and $87.7 \%$ and $42.8 \%$ for those in the dose-escalation group, respectively. The 5-year OS rates seemed higher in the high-dose group $(42.8 \%)$ than in the conventional-dose group (21\%), which showed a significant difference $(p=0.028)$. The estimated Kaplan-Meier curves for OS and RFS are shown in Figures 2 and 3 , respectively.

\section{Patterns of failure}

Table 2 depicts the rates and sites of the first recurrence. A total of 55 patients $(68.8 \%)$ experienced treatment failures, including locoregional failure (LRF) alone in 29 patients (36.3\%), distant metastasis alone in $19(23.8 \%)$, and combined LRF and distant metastasis in 7 (8.8\%); 25 (31.3\%) had no evidence of disease. The incidence of LRF was significantly lower in the control vs the observation group $(64.5 \%$ in the observation group vs $37.5 \%$ in the control group, respectively; $p=0.04)$. The distant failure rate $(25.8 \%$ vs $45.8 \%)$ between 2 groups did not reach statistical significance.

\section{Treatment-related toxicity}

The toxicities induced by CCRT were mainly skin reaction, esophagitis, hematologic toxicity, and radiation pneumonia. Treatment-induced toxicities for all patients are detailed in Table 3. No treatment-related deaths were found. As for hematological adverse events, events graded $\geq 3$ during CRT were seen in $18 / 43$ patients $(41.8 \%$ ) and $21 / 37$ patients $(56.7 \%)$, respectively. On the other hand, nonhematological side effects, radiation esophagitis, were seen at a high frequency. The incidences of grade $\geq 3$ esophagisis was higher in the control group than in the observation group $(8 / 37,21.6 \%$ vs. $4 / 43,9.3 \%)$. The rate of pneumontitis was $4(10.8 \%)$ in the standard-dose group, whereas it was $10(25.0 \%)$ in the dose-escalation group. No significant differences were observed between the 2 groups in the rates of other toxicities.

\section{Discussion}

The question of optimal radiation dose in the treatment of locally advanced EC has long been a topic of investigation. Since the establishment of 50.4 Gy as the standard dose in the management of locally advanced EC (RTOG 94-05), there have been multiple attempts at optimization of radiation dose.

A great number of studies were done to investigate the potential benefits of higher RT dose. The study of Zhang

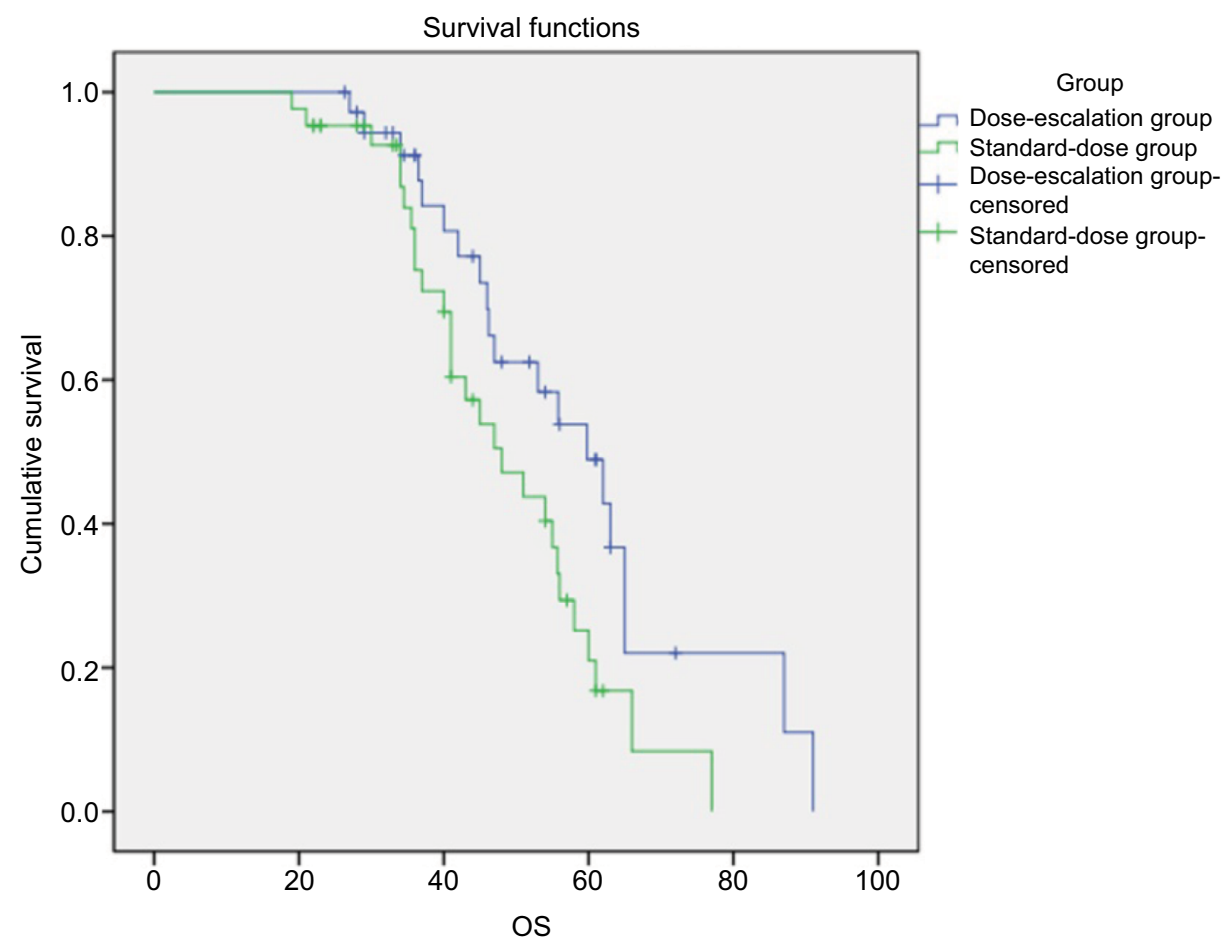

Figure 2 OS in the standard-dose group (group I: patients receiving CCRT <56 Gy) and dose-escalation group (group 2: patients receiving CCRT $\geq 59.4$ Gy) Note: $p=0.006$.

Abbreviations: OS, overall survival; CCRT, concurrent chemoradiotherapy. 


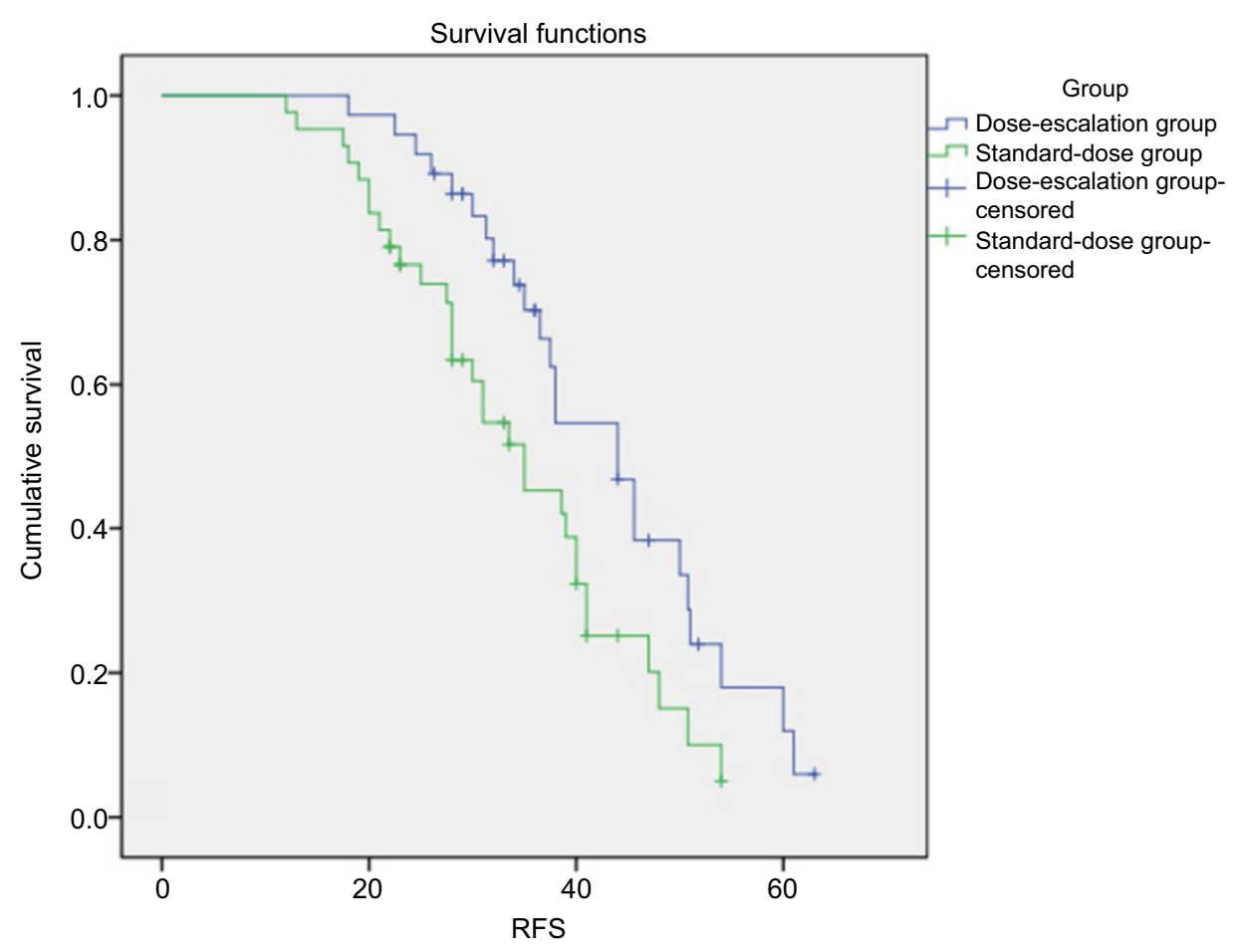

Figure 3 RFS in the standard-dose group (group I: patients receiving CCRT <56 Gy) and dose-escalation group (group 2: patients receiving CCRT $\geq 59.4$ Gy). Note: $p=0.019$.

Abbreviations: RFS, recurrence-free survival; CCRT, concurrent chemoradiotherapy.

Table 2 Failure status

\begin{tabular}{llll}
\hline Failure status & $\begin{array}{l}\text { Standard-dose } \\
\text { group, N (\%) }\end{array}$ & $\begin{array}{l}\text { Dose-escalation } \\
\text { group, N (\%) }\end{array}$ & p-value \\
\hline LRF only & $20(64.5 \%)$ & $9(37.5 \%)$ & 0.040 \\
DM only & $8(25.8 \%)$ & II (45.8\%) & 0.244 \\
Both LRF and DM & $3(9.7 \%)$ & $4(16.7 \%)$ & 0.698 \\
Total & $31 / 43$ & $24 / 37$ & \\
\hline
\end{tabular}

Abbreviations: LRF, local-regional failure; DM, distant metastasis.

Table 3 Treatment toxicity

\begin{tabular}{llll}
\hline Toxicity & $\begin{array}{l}\text { Standard- } \\
\text { dose } \\
\text { group }\end{array}$ & $\begin{array}{l}\text { Dose- } \\
\text { escalation } \\
\text { group }\end{array}$ & -value \\
\hline Skin reaction $(\geq 2)$ & 4 & 5 & 0.597 \\
Esophagitis $(\geq 3)$ & 4 & 8 & 0.124 \\
Pneumontitis $(\geq 3)$ & 4 & 10 & 0.037 \\
Hematologic toxicity $(\geq 3)$ & 18 & 21 & 0.184 \\
\hline
\end{tabular}

et $\mathrm{al}^{8}$ found that patients in the higher dose group ( $>51 \mathrm{~Gy}$ ) had a better 3-year local control rate (36\% vs $19 \%$ ) and a disease-free survival rate $(25 \%$ vs $10 \%)$ than those in the low-dose group ( $\leq 51 \mathrm{~Gy})$. No significant differences were found between high- and low-dose groups in OS $(p=0.054) .{ }^{8}$ A study was conducted by Suh et $\mathrm{al}^{10}$ to compare the prog- nosis between the 2 groups (the patients received a dose of $\leq 60$ or $>60 \mathrm{~Gy}) .{ }^{10}$ The high-dose group experienced a significantly lower LRF rate ( $32 \%$ vs $69 \%, p<0.01)$. However, no significant difference was noted between the 2 groups regarding $\mathrm{OS}(p=0.26)$. In line with these findings, the results from a study by $\mathrm{He}$ et $\mathrm{al}^{15}$ showed that the high-dose group had a significantly lower local failure rate $(17.9 \%$ vs $34.3 \%$, $p=0.024)$ than the low-dose group, but the OS was not significantly different $(p=0.617)$. However, some studies claimed that high-dose radiotherapy may provide a survival benefit. In the retrospective analysis by Kim et al, ${ }^{16}$ the correlation between radiation dose and OS for EC patients treated with definitive CRT was investigated. The results suggested that patients who underwent high-dose radiotherapy had significantly better survival than patients receiving $<60 \mathrm{~Gy}$ RT when treated with concurrent chemotherapy. Similarly, a study conducted by Chen et $\mathrm{a}^{17}$ found that a dose higher than that used for standard radiotherapy may lead to better survival for ESCC patients undergoing CCRT. These conflicting results revealed that the optimal definitive radiation dose should be managed on an individual basis.

Patients with ESCC receiving CRT seem to show a disparity in treatment response. For the radiosensitive EC, 
dose-escalation radiotherapy may provide survival benefit. Conversely, it is very difficult to confer a benefit to a radiation-resistant patient, and dose escalation even may lead to increase the treatment-related toxicities. At present, data on the survival outcomes and recurrence patterns of patients with $\mathrm{EC}$ who achieved a $\mathrm{CR}$ after standard-dose radiotherapy remain scarce. In this study, we investigated the survival outcomes and recurrence patterns in patients who achieve cCR after standard radiation dose radiotherapy. Furthermore, we also explored whether the higher radiation dose therapy could confer benefits in local control or OS in those patients.

To the best of our knowledge, this is the first retrospective analysis on whether the higher radiation dose could confer benefits for EC patients who achieved a CR after standarddose radiotherapy (50.4 Gy). There are 2 principal findings in this study. First, we demonstrated that at least $60 \%$ of ESCC patients who achieve cCR after CRT developed disease recurrences, with LR being the predominant failure pattern. However, the LR rate in the control group was significantly lower than that in the standard-dose group. Second, as shown in Figure 2, the survival time and the RFS in the control group were longer than in the observation group, which were statistically different $(p<0.05)$. The results of the current study suggest that the subgroup of patients who underwent dose escalation had significantly better LC and OS than patients receiving 50.4 Gy when treated with concurrent chemotherapy. Furthermore, the CRT-induced toxicities were also acceptable in the dose-escalation group.

In this scenario, it is our institutional policy to perform a dose-escalation radiotherapy aimed at achieving a better tumor control. Unfortunately, LR rates remained suboptimal (37.5\%) even after dose-escalation radiotherapy. Such rates are significantly higher than those identified $(<10 \%)$ in patients who underwent resection. ${ }^{18-20}$ McLoughlin et a ${ }^{21}$ suggested that after chemoradiation, cCR was not substantially predictive of pathological CR. Similarly, a study conducted by Stiles et $\mathrm{al}^{22}$ also revealed that a CR on posttreatment PET scan predicts but should not be assumed to be synonymous with complete pathologic response in EC patients. Accordingly, only $23 \%-40 \%$ of cCR patients actually have no residual cancer on pathological examination. ${ }^{23-25}$ In summary, although the lesion has reached clinical remission after CRT, there may still be residual lesions in the primary site. Therefore, dose-escalation radiotherapy may eliminate the residual lesion and improve the local control and OS.

This study has a few limitations that need to be considered when interpreting the results. The study is limited by its retrospective nature, and we cannot account for potential selection bias, which may limit the generalizability of our results. Second, our sample size is small, and further independent studies in larger populations are needed to confirm and validate our results. Finally, it is possible that treatmentrelated toxicities were underestimated due to the study's retrospective setting.

\section{Conclusion}

Taken together, our results indicate that dose-escalated radiotherapy yields more favorable survival outcomes in EC patients who achieved a cCR after standard-dose radiotherapy. Further independent studies in larger populations are needed to confirm and validate our results.

\section{Disclosure}

The authors report no conflicts of interest in this work.

\section{References}

1. Torre LA, Bray F, Siegel RL, Ferlay J, Lortet-Tieulent J, Jemal A. Global cancer statistics, 2012. CA Cancer J Clin. 2015;65(2):87-108.

2. Ferlay J, Shin HR, Bray F, Forman D, Mathers C, Parkin DM. Estimates of worldwide burden of cancer in 2008: GLOBOCAN 2008. Int $J$ Cancer. 2010;127(12):2893-2917.

3. Wheeler JB, Reed CE. Epidemiology of esophageal cancer. Surg Clin North Am. 2012;92(5):1077-1087.

4. Shahbaz SCM, Luketich JD, Landreneau RJ, Abbas G. Esophageal cancer: an update. Int J Surg. 2010;8(6):417-422.

5. Cooper JS, Guo MD, Herskovic A, et al. Chemoradiotherapy of locally advanced esophageal cancer: long-term follow-up of a prospective randomized trial (RTOG 85-01). Radiation Therapy Oncology Group. JAMA. 1999;281(17):1623-1627.

6. Yen YC, Chang JH, Lin WC, et al. Effectiveness of esophagectomy in patients with thoracic esophageal squamous cell carcinoma receiving definitive radiotherapy or concurrent chemoradiotherapy through intensity-modulated radiation therapy techniques. Cancer. 2017;123(11):2043-2053.

7. Minsky BD, Pajak TF, Ginsberg RJ, et al. INT 0123 (Radiation Therapy Oncology Group 94-05) phase III trial of combined-modality therapy for esophageal cancer: high-dose versus standard-dose radiation therapy. J Clin Oncol. 2002;20(5):1167-1174.

8. Zhang Z, Liao Z, Jin J, et al. Dose-response relationship in locoregional control for patients with stage II-III esophageal cancer treated with concurrent chemotherapy and radiotherapy. Int J Radiat Oncol Biol Phys. 2005;61(3):656-664.

9. Seung SK, Smith JW, Molendyk J, et al. Selective dose escalation of chemoradiotherapy for esophageal cancer: role of treatment intensification. Semin Oncol. 2004;31(6 Suppl 18):13-19.

10. Suh YG, Lee IJ, Koom WS, et al. High-dose versus standard-dose radiotherapy with concurrent chemotherapy in stages II-III esophageal cancer. Jpn J Clin Oncol. 2014;44(6):534-540.

11. Ma J, Wang $Z$, Wang $C$, et al. Individualized radiation dose escalation based on the decrease in tumor FDG uptake and normal tissue constraints improve survival in patients with esophageal carcinoma. Technol Cancer Res Treat. 2017;16(1):75-80.

12. Di Fiore F, Lecleire S, Rigal O, et al. Predictive factors of survival in patients treated with definitive chemoradiotherapy for squamous cell esophageal carcinoma. World J Gastroenterol. 2006;12(26):4185-4190. 
13. Yamashita H, Okuma K, Wakui R, Kobayashi-Shibata S, Ohtomo K, Nakagawa K. Details of recurrence sites after elective nodal irradiation (ENI) using 3D-conformal radiotherapy (3D-CRT) combined with chemotherapy for thoracic esophageal squamous cell carcinoma - a retrospective analysis. Radiother Oncol. 2011;98(2):255-260.

14. Onozawa M, Nihei $\mathrm{K}$, Ishikura $\mathrm{S}$, et al. Elective nodal irradiation (ENI) in definitive chemoradiotherapy (CRT) for squamous cell carcinoma of the thoracic esophagus. Radiother Oncol. 2009;92(2): 266-269.

15. He L, Allen PK, Potter A, et al. Re-evaluating the optimal radiation dose for definitive chemoradiotherapy for esophageal squamous cell carcinoma. J Thorac Oncol. 2014;9(9):1398-1405.

16. Kim HJ, Suh YG, Lee YC, et al. Dose-response relationship between radiation dose and loco-regional control in patients with stage II-III esophageal cancer treated with definitive chemoradiotherapy. Cancer Res Treat. 2017;49(3):669-677.

17. Chen CY, Li CC, Chien CR. Does higher radiation dose lead to better outcome for non-operated localized esophageal squamous cell carcinoma patients who received concurrent chemoradiotherapy? A population based propensity-score matched analysis. Radiother Oncol. 2016;120(1):136-139.

18. Sudo K, Taketa T, Correa AM, et al. Locoregional failure rate after preoperative chemoradiation of esophageal adenocarcinoma and the outcomes of salvage strategies. J Clin Oncol. 2013;31(34):4306-4310.
19. Rohatgi PR, Swisher SG, Correa AM, et al. Failure patterns correlate with the proportion of residual carcinoma after preoperative chemoradiotherapy for carcinoma of the esophagus. Cancer. 2005;104(7):1349-1355.

20. Smit JK, Güler S, Beukema JC, et al. Different recurrence pattern after neoadjuvant chemoradiotherapy compared to surgery alone in esophageal cancer patients. Ann Surg Oncol. 2013;20(12):4008-4015.

21. McLoughlin JM, Melis M, Siegel EM, et al. Are patients with esophageal cancer who become PET negative after neoadjuvant chemoradiation free of cancer. J Am Coll Surg. 2008;206(5):879-886; discussion 886-887.

22. Stiles BM, Salzler G, Jorgensen A, et al. Complete metabolic response is not uniformly predictive of complete pathologic response after induction therapy for esophageal cancer. Ann Thorac Surg. 2013;96(5):1820-1825.

23. Schneider PM, Metzger R, Schaefer H, et al. Response evaluation by endoscopy, rebiopsy, and endoscopic ultrasound does not accurately predict histopathologic regression after neoadjuvant chemoradiation for esophageal cancer. Ann Surg. 2008;248(6):902-908.

24. Sarkaria IS, Rizk NP, Bains MS, et al. Post-treatment endoscopic biopsy is a poor-predictor of pathologic response in patients undergoing chemoradiation therapy for esophageal cancer. Ann Surg. 2009;249(5):764-767.

25. Kim MP, Correa AM, Lee J, et al. Pathologic T0N1 esophageal cancer after neoadjuvant therapy and surgery: an orphan status. Ann Thorac Surg. 2010;90(3):884-890; discussion 890-891.
Cancer Management and Research

\section{Publish your work in this journal}

Cancer Management and Research is an international, peer-reviewed open access journal focusing on cancer research and the optimal use of preventative and integrated treatment interventions to achieve improved outcomes, enhanced survival and quality of life for the cancer patient. The manuscript management system is completely online and includes

\section{Dovepress}

a very quick and fair peer-review system, which is all easy to use. Visit http://www.dovepress.com/testimonials.php to read real quotes from published authors. 